\title{
Open Social Learner Models for Self-Regulated Learning and Learning Motivation
}

\author{
Julio Guerra* \\ School of Information Sciences \\ University of Pittsburgh \\ jdg60@pitt.edu
}

\begin{abstract}
Open Learner Models (OLM) have demonstrated a multitude of benefits supporting metacognition and engaging learners. Although researchers have study different representations of OLM, a broader view that situates OLM in Self-Regulated Learning (SRL) is missing. An important element in SRL that can bring a better understanding of these tools and their effects concerns to learning motivation theories. In this work I connect these aspects and propose to study the effects of OLM and motivational factors drawn from learning motivation theories. To account for a broader spectrum of OLM representations, I proposed to explore the addition of social information and different levels of granularity in the OLM. I propose to evaluate different designs and then to evaluate the resulting interface in field studies. With the proposed work I expect to gain a deeper understanding of the effects of OLM tools which can be used to guide the development of better tools, better personalization and adaptive mechanisms, better use of such tools in supporting Self-Regulated Learning, and ultimately impact positively in learning.
\end{abstract}

\section{CCS Concepts}

•Human-centered computing $\rightarrow$ Empirical studies in visualization;

\section{Keywords}

Open Learner Model, Learning Motivation

\section{INTRODUCTION}

Open learner models (OLMs) are learning tools that take an internal model of the learner maintained by a computerbased adaptive or tutoring system and shows it to the learner. According to [7], OLMs can support a variety of aspects, including metacognitive processes such as awareness and

*Advisor: Peter Brusilovsky, School of Information Sciences, University of Pittsburgh

Permission to make digital or hard copies of part or all of this work for personal or classroom use is granted without fee provided that copies are not made or distributed for profit or commercial advantage and that copies bear this notice and the full citation on the first page. Copyrights for third-party components of this work must be honored. For all other uses, contact the owner/author(s).

UMAP '16 July 13-17, 2016, Halifax, NS, Canada

(C) 2016 Copyright held by the owner/author(s).

ACM ISBN 978-1-4503-4370-1/16/07.

DOI: http://dx.doi.org/10.1145/2930238.2930375 learning control, and collaboration and trust in the system, among others. Previous work on open learner models (OLMs) and our previous work on open social learner models (OSLMs) [6, 13] show interesting effects on engagement with the learning content and the system, and sometimes contradictory guidance effects. For example [22, 17] showed that OLM helps learners to select better problems and [14] shows that OSLMs produce a "conservative guidance" effect that makes students advance more sequentially through the course content. Moreover, few articles report on the effects of OLMs on learning [18, 22], but those that do note that they are usually buffered by metacognitive aspects [9]. I believe that the effects of OLMs are hidden behind individual differences. For example, [22] found that OLMs produced significant positive differences between pretest and posttest only for "less able" students. In our own work, we have found evidence that OSLMs produce different effects across gender [6]. On the other hand, research on learning theories connects the engagement found in self-regulated learning with different aspects, which range from metacognitive skills [2], to learner beliefs [11], to goal orientation [28], among others. For example, literature on learning motivation connects to self-regulated learning and finds evidence to suggest that motivational factors explain the engagement in SRL processes [29, 28]; this suggests that learning motivation should be considered in the study of SRL environments, such as OLMs. However, no study to date has explored the effects of OLMs, OSLMs, and learning motivation together, and an overall study of the relationships between engagement in using OLMs and SRLs is missing. Achieving a better understanding of the effects that OLM tools produce in learners is important, because it can guide the development of better tools, better personalization, and adaptive mechanisms, which will allow us to make better use of such tools in supporting self-regulated learning processes.

In this work, I propose to study the effects of using an OLM in conjunction with a variety of motivational factors, as defined by different learning motivation theories and frameworks, including the achievement-goal orientation framework [12], self-theories [11], and learning activation [23]. An OSLM system already deployed in our previous studies will be used [13] (see Figure 1). This system incorporates social comparison features that have demonstrated engagement effects in previous studies $[6,13]$. To better study the effects of this system and the effect of the OLM, I propose to implement and evaluate different visualizations that complement the information displayed by the system, with the addition of a fine-grained learner model. A fine-grained learner model 
represents the level of inferred knowledge of the learner in the multitude of conceptual units of the domain and their relationships, which gives the learner a potential powerful metacognitive tool [9], and enables my work to study the effects of granularity and structure on the OLM.

\section{RELATED WORK}

In traditional adaptive and personalized computed-based learning environments, a user model captures individual aspects, preferences, and general aspects of the learning progress of a student, which allows the system to perform both adaptation and personalization tasks [5]. Open learner models (or open student models), OLMs, release a representation of the user model to the learner with the goal of promoting reflection and encouraging self-regulated processes [7].

Different types of OLMs have been explored, and [8] offers a review of these. Researchers have explored different representations that range from overall knowledge states skillmeters [22] to structured representations such as treemaps [4] and concept-maps [18]. Some works present systems with different alternative or complementary representations of their users, such as prerequisite-based concept-maps, hierarchical representation of concept details, and hierarchical representation of the overall course organization [20].

The review of [8] also distinguish different approaches that incorporate a social dimension into an OLM. There have been studies on group interaction modeling, where the learners' interactions are represented to support collaboration and assessment of collaborative work [27]. Another approach explores awareness, social navigational support, and social-comparison effects as a result of showing the models of other learners or aggregated knowledge/progress states of groups of learners [3,6], which has been called an Open Social Learner Model (OSLM), or an Open Social Student Model (OSSM). OSLM has been demonstrated to produce different effects in both engagement and navigational patterns. For example $[15,16]$ in different studies consistently found that by showing the models of peer learners, students covered more topics in the system, reached higher success rates in self-assessment problems, and that strong students lead in system exploration. Our later work confirms these findings and reveals some other effects. For example, in [6], we showed how the treatment group, which was exposed to social comparison visualizations, presents higher rates of system usage, learning effectiveness (see [25] for a detailed description of learning or instructional effectiveness), and interaction effects with gender. While we have repeatedly demonstrated the positive uses of an OSLM in classroom studies, our past work in OSLM explores a relatively simple visualization of the learner progress in a coarse-grained representation that is based on topics (see Figure 1). My work focuses on taking this exploration further, and to study the effects of an OSLM that combines both coarse and finegrained representations.

\section{WHY LEARNING MOTIVATION?}

Self-regulated learning (SRL) defines an active learner who monitors and controls their own learning process cognitively, meta-cognitively, and emotionally [29]. Zimmerman summarizes three dimensions of SRL: (i) the dual focus in selfregulation process and strategies that target those processes; (ii) the key role of continuing feedback that enables SRL to

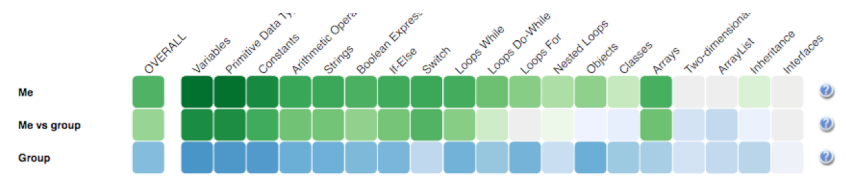

Figure 1: Mastery Grids with added social comparison features. the first row represents the progress of the learner (each cell is a topic), the third row represents the average progress of the active students, and the second row represents the difference between the learner and the rest of the class.

happen; and (iii) the interdependence between motivation and self-regulating processes [29]. This interdependence has been broadly studied. For example, the social cognitive view of SRL focuses in self-efficacy, a measure of self-regulation, as the force behind motivation [2]. Other authors have confirmed the positive relation between self-efficacy and other motivational elements, like goal-setting [26]. Moreover, selfregulated learners "who proactively seek out information when needed and take the necessary steps to master it" [29], are closely related to the Mastery-Approach orientation defined by the achievement-goal orientation framework [12]. This framework contains four goal orientations: MasteryApproach, Mastery-Avoidance, Performance-Approach, and Performance-Avoidance. Mastery-Approach oriented students pursue learning, while Performance-oriented students pursue the demonstration of performance and are usually more sensitive to comparison and scores. Mastery-Avoidance students avoid achieving the minimum, and PerformanceAvoidance students avoid performing worse than others or receiving the lowest scores. The implications of these orientations in regulatory learning processes have been well reported in existing literature. For example, according to [28], the goal orientation adopted by the learner is a positive predictor of adaptive behaviors: mastery-oriented students present higher levels of SRL elements, including higher self-efficacy and the use of self-regulatory strategies. Researchers have studied the factors that can foster different achievement-goal orientations. Self-theories [11] reveal how the learners' beliefs in their own intelligence as a fixed or growable resource impact in engagement in adaptive behaviors and learning success. When facing challenging tasks, students who consider intelligence to be a fixed capacity tend to feel threatened, become especially sensitive to external judgment, and usually embrace performance goals. On the other hand, growth-minded students who believe that intelligence can always grow tend to take learning challenges positively, expend more effort, and assume mastery-oriented goals [21]. External factors also impact a student's orientation. For example, mastery-oriented environmental factors, such as an environment that supports autonomous work, can foster the adoption of a Mastery orientation [10], while performance-oriented elements can account for the adoption of performance goals [24]. Research has also established relationships between the different goal orientations. For example, a student can present high levels of performance and mastery orientation goals at the same time [1]. These elements are important to my work because they provide a basis for the idea of incorporating performance and mastery oriented features together in the system, such as social comparison and a fine-grained representation of the OLM, and 
also suggests that such features can support the simultaneous adoption of both mastery and performance orientations.

\section{PROPOSED RESEARCH}

I propose to study the relationship between the effects of using an OLM and the learning motivation profile of the learners. This relationship has two major aspects. On one hand, the motivational profile, as defined by different motivational theories and frameworks, is hypothesized to explain different engagement approaches. On the other hand, the motivational profile is not static and can be influenced by the learning experience. By knowing how the motivational profile expresses itself in the patterns of usage of the system, we can see if these patterns change by using different representations of OLM and OSLM with different levels of granularity, and the ways in which these variants might affect overall changes in motivation. My work seeks to answer the following research questions.

- How do students with different motivational profiles engage in using a learning system that provides an OLM?

- What are the effects of social comparison features?

- What are the effects of the granularity of the OLM?

- How does an OLM influence motivation?

To answer these questions, I have organized the proposed work in two parts. Part 1 explores and assesses alternatives to represent an OLM. The goal is to reduce the design space of a OLM and to inform the development of a definitive interface, which I call the Rich-OLM. We foresee that a Rich-OLM will involve both a coarse-grained and a finegrained view of the learner model, along with the inclusion of aggregated information from other learners. The finegrained view will visualize concepts, as well as the structure of relationships between concepts and the coarse elements (topics). We think that all of this information can support students with different goals and motivations when using the system. A design study is proposed to carry the evaluation of different design alternatives. The resulting Rich-OLM is evaluated in Part 2 through term-long classroom (field) studies to assess its overall effect on the learning experience, which includes a variety of aspects: system usage, navigational and activity patterns, changes in motivational factors, and learning outcomes.

The motivational profile is measured in Part 2. The factors included in the motivational profile are distilled from different learning motivation theories, including the Learning Activation Questionnaire [23]. the Achievement Goal Revised questionnaire [12] and the Mindset questionnaire [11]. Learning Activation measures the factors of Fascination, Values, and Competency-Beliefs with 14 items, all of them being domain-dependent, and using 4 and 5 points of ordinal scales. The Achievement Goal questionnaire contains 12 questions, 3 for each factor: Mastery-Approach, Mastery-Avoidance, Performance-Approach, and PerformanceAvoidance. All questions use a 7-point Likert scale. The Mindset questionnaire contains 12 questions using a 6-point Likert scale with 6 for each of the opposite factors: an Incremental mindset (the intelligence is growable) and an Entity mindset (the intelligence is fixed). The motivation questionnaires will be given at both the beginning and at the end of the classroom studies, which wil allow for the measure of any change in the motivational profile of the students.
Table 1: Number of active students (those who used the system) in previous classroom studies.

\begin{tabular}{lcc}
\hline Domain & Active Users & Questionnaires collected \\
\hline Java & 217 & 162 \\
SQL & 86 & 80 \\
Python & 490 & 426 \\
\hline
\end{tabular}

\section{CURRENT PROGRESS}

To this date, we have conducted several classroom studies using Mastery Grids OSLM (Figure 1); the results of some of these are reported in different articles [19, 6, 13]. As a result, a considerable amount of data on the usage of the system has been collected, including questionnaires measuring the motivational profile. Data has been collected in three domains: Java programming, SQL programming, and Python programming, in different class groups using Mastery Grids with varied material that includes assessment exercises, code examples, and code animations. Table 1 shows the number of active students and students who have answered the questionnaires in previous studies. Current classroom studies are being conducted in a "pure" baseline mode, without the use of the OLM interface. New term-long classroom studies in the 3 domains (Java, SQL, and Python programming) are planned for the second part of 2016, where an extended version of Mastery Grids, including fine-grained OSLM features, will be tested.

In parallel, Part 1 is being executed. We are exploring different alternatives of complementing Mastery Grids with a view of the fine-grained learner model; namely, the concept space representation of the learner model. A set of interviews were conducted with students who used Mastery Grids in previous terms with the goal of verifying the extent to which students use the system differently and the extent to which they think that a detailed OLM, including a fine-grained view (concept space), and the exposure of the models of other learners are valuable information to support their learning and influences their motivation to use the system. Results confirm these views: students have differing opinions about what information is helpful and how they would eventually use that information. In general, students would like to be able to see the concept space (which concepts are in each topic) and how they are doing in learning those concepts. Second, they would like to know how others are doing, but they also expressed some concerns about this information: What does it mean for a student to be in the average of the class? What if other students who do a lot of activities are advanced students that need to expend only a little effort to excel? Addressing these concerns is necessary in order to generate a visualization that can support a variety of students.

\section{EXPECTED CONTRIBUTIONS}

We expect to achieve the following main contributions:

- Role of motivational factors in engagement with SRL learning tool.

- Design guidelines for OLM that consider different motivational profiles.

- Understanding role of the different features in an OLM, including granularity and social comparison features.

- The effects of using OLMs on a change of motivation. 
- Adaptation guidelines for adaptive navigational support systems, based on OLMs.

\section{REFERENCES}

[1] C. Ames. Classrooms: Goals, structures, and student motivation. Journal of educational psychology, 84(3):261, 1992.

[2] A. Bandura. Social foundations of thought and action: A social cognitive theory. Prentice-Hall, Inc, 1986.

[3] P. Brusilovsky, G. Chavan, and R. Farzan. Social adaptive navigation support for open corpus electronic textbooks. In Adaptive Hypermedia and Adaptive Web-Based Systems, pages 24-33. Springer, 2004.

[4] P. Brusilovsky, I.-H. Hsiao, and Y. Folajimi. Quizmap: open social student modeling and adaptive navigation support with treemaps. In Towards Ubiquitous Learning, pages 71-82. Springer, 2011.

[5] P. Brusilovsky and E. Millán. User models for adaptive hypermedia and adaptive educational systems. In The adaptive web, pages 3-53. Springer-Verlag, 2007.

[6] P. Brusilovsky, S. Somyurek, J. Guerra, R. Hosseini, V. Zadorozhny, and P. Durlach. Open social student modeling for personalized learning. IEEE Transactions on Emerging Topics in Computing, PP(99):1-1, 2015.

[7] S. Bull and J. Kay. Student models that invite the learner in: The smili:() open learner modelling framework. International Journal of Artificial Intelligence in Education, 17(2):89-120, 2007.

[8] S. Bull and J. Kay. Open learner models. In Advances in intelligent tutoring systems, pages 301-322. Springer, 2010.

[9] S. Bull and J. Kay. Open learner models as drivers for metacognitive processes. In International Handbook of Metacognition and Learning Technologies, pages 349-365. Springer, 2013.

[10] K. D. Ciani, M. J. Middleton, J. J. Summers, and K. M. Sheldon. Buffering against performance classroom goal structures: The importance of autonomy support and classroom community. Contemporary Educational Psychology, 35(1):88-99, 2010.

[11] C. S. Dweck. Self-theories: Their role in motivation, personality, and development. Psychology Press, 2000.

[12] A. J. Elliot and K. Murayama. On the measurement of achievement goals: Critique, illustration, and application. Journal of Educational Psychology, 100(3):613, 2008.

[13] J. Guerra, R. Hosseini, S. Somyürek, and P. Brusilovsky. An intelligent interface for learning content: Combining an open learner model and social comparison to support self-regulated learning and engagement. IUI, 2016.

[14] R. Hosseini, I.-H. Hsiao, J. Guerra, and P. Brusilovsky. What should i do next? adaptive sequencing in the context of open social student modeling. In Design for Teaching and Learning in a Networked World, pages 155-168. Springer, 2015.

[15] I.-H. Hsiao, F. Bakalov, P. Brusilovsky, and B. König-Ries. Progressor: social navigation support through open social student modeling. New Review of Hypermedia and Multimedia, 19(2):112-131, 2013.
[16] I.-H. Hsiao and P. Brusilovsky. Motivational social visualizations for personalized e-learning. In 21st Century Learning for 21st Century Skills, pages 153-165. Springer, 2012.

[17] I.-H. Hsiao, S. Sosnovsky, and P. Brusilovsky. Guiding students to the right questions: adaptive navigation support in an e-learning system for java programming. Journal of Computer Assisted Learning, 26(4):270-283, 2010.

[18] A. Kumar and A. Maries. The effect of open student model on learning: A study. FRONTIERS IN ARTIFICIAL INTELLIGENCE AND APPLICATIONS, 158:596, 2007.

[19] T. D. Loboda, J. Guerra, R. Hosseini, and P. Brusilovsky. Mastery grids: An open source social educational progress visualization. In Open Learning and Teaching in Educational Communities, pages 235-248. Springer, 2014.

[20] A. Mabbott and S. Bull. Student preferences for editing, persuading, and negotiating the open learner model. In Intelligent tutoring systems, pages 481-490. Springer, 2006.

[21] J. A. Mangels, B. Butterfield, J. Lamb, C. Good, and C. S. Dweck. Why do beliefs about intelligence influence learning success? a social cognitive neuroscience model. Social cognitive and affective neuroscience, 1(2):75-86, 2006.

[22] A. Mitrovic. Evaluating the effect of open student models on self-assessment. In International Journal of Artificial Intelligence in Education. Citeseer, 2007.

[23] D. W. Moore, M. E. Bathgate, J. Chung, and M. A. Cannady. Technical report: Measuring activation and engagement. Technical report, Activation Lab, Learning Research and Development Center, University of Pittsburgh, Pittsburgh, PA (USA), 2011.

[24] P. A. O'Keefe, A. Ben-Eliyahu, and L. Linnenbrink-Garcia. Shaping achievement goal orientations in a mastery-structured environment and concomitant changes in related contingencies of self-worth. Motivation and Emotion, 37(1):50-64, 2013.

[25] F. G. Paas and J. J. Van Merriënboer. The efficiency of instructional conditions: An approach to combine mental effort and performance measures. Human Factors: The Journal of the Human Factors and Ergonomics Society, 35(4):737-743, 1993.

[26] D. H. Schunk. Goal setting and self-efficacy during self-regulated learning. Educational psychologist, 25(1):71-86, 1990.

[27] K. Upton and J. Kay. Narcissus: group and individual models to support small group work. In User Modeling, Adaptation, and Personalization, pages 54-65. Springer, 2009.

[28] C. A. Wolters, L. Y. Shirley, and P. R. Pintrich. The relation between goal orientation and students' motivational beliefs and self-regulated learning. Learning and individual differences, 8(3):211-238, 1996.

[29] B. J. Zimmerman. Self-regulated learning and academic achievement: An overview. Educational psychologist, 25(1):3-17, 1990. 